NBSIR 78-1552

\title{
The Influence of Scattering Foils on Absorbed Dose Distributions From Electron Beams
}

M. J. Berger and S. M. Seltzer

Radiation Physics Division

Center for Radiation Research National Measurement Laboratory

National Bureau of Standards

Washington, D.C. 20234

November 1978

Prepared for

Office of Naval Research

Arlington, Virginia 22217

Department of Energy

Washington, D.C. 20545

National Cancer Institute

National Institutes of Health

Bethesda, Maryland 20014 
NBSIR 78-1552

THE INFLUENCE OF SCATTERING FOILS ON ABSORBED DOSE DISTRIBUTION FROM ELECTRON BEAMS

M. J. Berger and S. M. Seltzer

Radiation Physics Division Center for Radiation Research National Measurement Laboratory National Bureau of Standards Washington, D.C. 20234

November 1978

Prepared for Office of Naval Research Arlington, Virginia 22217

Department of Energy

Washington, D.C. 20545

National Cancer Institute

National Institutes of Health

Bethesda, Maryland 20014

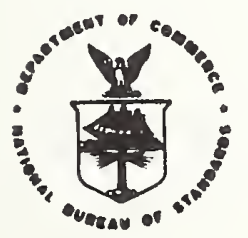

U.S. DEPARTMENT OF COMMERCE, Juanita M. Krops, Secrotary Dr. Sidnoy Harman. Under Secrotary Jordan J. Banuch. Assistant Socrotary for Scionce and Technology NATIONAL BUREAU OF STANDARDS. Emost Ambler, Director 


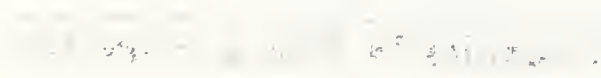

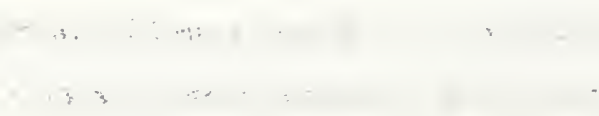


The Influence of Scattering Foils on Absorbed Dose Distributions from Electron Beams*

\author{
M. J. Berger and S. M. Seltzer \\ Center for Radiation Research \\ National Bureau of Standards \\ Washington, D.C. 20234
}

This paper describes a calculation of the spatial distribution of absorbed dose in a water phantom irradiated by electron beams with energies from 5 to $40 \mathrm{MeV}$. It is assumed that the initially monoenergetic, monodirectional and narrowly collimated electron beam passes through a lead scattering foil and $100 \mathrm{~cm}$ of air before reaching the water phantom. In the first part of the work a calculation is made of the energy spread and angular diffusion of the beam due to its passage through the scattering foil and air. In the second part of the work, a calculation is made of the penetration of the modified beam into the phantom. The second calculation takes into account the limitation of the beam to finite field size at the surface of the phantom, and the contribution of secondary bremsstrahlung generated in the scattering foil to the absorbed dose in the phantom.

*Based on a talk presented at the Symposium "Dosimetry and Treatment Planning Using High Energy Electrons" at the 14'th International Congress of Radiology, Rio de Janeiro, Brazil, October 23-29, 1977. 


\section{Introduction}

Various techniques are available for calculating the penetration and diffusion of electrons in extended media. Among these, the most widely used are the moments method ${ }^{1-5}$ and the Monte Carlo method ${ }^{6-10}$. The computational schemes and computer codes based on these methods have been elaborated to the point where they rather accurately take into account all the pertinent processes, including the angular deflections of electrons in elastic Coulomb collisions with atoms, angular deflections and energy losses resulting from inelastic Coulomb collisions with atomic electrons, the setting in motion of secondary electrons in ionization events, and the emission of secondary bremsstrahlung quanta.

Whereas the physical processes have been treated in considerable detail, the boundary conditions assumed in the electron transport calculations made so far have been rather oversimplified. It has usually been assumed that monoenergetic and monodirectional beams of electrons are incident perpendicularly onto the medium. Moreover, broad-beam conditions have often been assumed, so the the calculations were limited to the determination of onedimensional depth-dose distributions.

The conditions of irradiation in radiation therapy and in industrial applications are more complex. Even though the electron beams from the accelerator may initially be highly monoenergetic and monodirectional, they must pass through various materials (beam-spreading foils, monitors, air, etc.) on their way to the medium (phantom, patient). Upon entry into the medium the beams have therefore acquired a significant energy spread and angular divergence. These beam modifications, and the collimation of the beam to finite field size, have a considerable influence on the spatial 
distribution of absorbed dose within the phantom ${ }^{1-19}$. We have attempted to extend the electron transport calculations in order to take some of these complications into account. In particular, we have considered the effect of lead beam-spreading foils, and the effect of collimation to finite field size. The arrangement of the scattering foil, collimator and water phantom assumed in our calculations is indicated in Fig. 1. The parameters of the problem are: the electron beam energy, the material and thickness of the foil, the distance $s$ between foil and phantom, the radius $r_{F}$ of the (circular) field irradiated by the beam, and the depth $z$ and radial distance $\rho$ from the beam axis to the point where the absorbed dose is to be determined. Among the various calculational techniques that are available, the Monte Carlo method, i.e. the simulation of particle tracks with the use of random numbers, has the greatest flexibility. Through the addition of complex-geometry routines, the Monte Carlo method could be extended to treat electron transport in the scattering foil and in the phantom in a single integrated calculation. However, this would require a very large amount of numerical computation, and it seems hardly practical to make lengthy Monte Carlo calculations for each of the many foil-collimator configurations that may be of interest.

We therefore adopted an alternative approach, which is computationally less demanding. The use of the Monte Carlo method was limited to the treatment of two separate restricted transport problems: (a) the transmission of electrons through foils, and (b) the penetration of monoenergetic, pointmonodirectional electron beams into a water phantom. The results from calculations (a) and (b) were then combined according to a superposition rule to obtain absorbed-dose distributions for various configurations of interest. 


\section{Foil Transmission}

In the foil transmission calculations, a monoenergetic, point-monodirectional beam of electrons was assumed to be incident perpendicularly on the scattering foil. Calculations were made for eight typical cases, involving lead scattering foils with thicknesses between $0.1 \mathrm{~mm}$ and $0.6 \mathrm{~mm}$, and beam energies from $5 \mathrm{MeV}$ to $40 \mathrm{MeV}$. The output of the calculations included the distribution in energy and angle of the transmitted electrons and secondary bremsstrahlung photons. The lateral spatial multiple scattering deflections of electrons and photons in the foil are negligibly small so that the radiation can be assumed to emerge from an effective focal point on the beam axis at the exit surface of the foil.

The modification of the electron and photon beams during their passage through the air between the lead foil and the phantom was treated by an approximate method. Monte Carlo transmission calculations were actually done for a two-layer medium consisting of a lead foil followed by a layer of condensed air-equivalent material. The electrons and photons emerging from the second layer were assumed to travel to the phantom without further scattering. Inasmuch as the passage through the air has a strong effect only on the spectra but not on the angular distribution of the transmitted radiation, we expect that the error incurred by this approximate schematization is smal1.

For the thin foils considered here, the correlation between the energy and direction of the transmitted radiation is quite small, expecially for deflection angles up to $20^{\circ}$. Therefore the calculated distributions can be accurately represented as the product of two one-variable functions: an energy spectrum, and an angular distribution. These two quantities are shown in Figs. 2 to 6 , which pertain to the transmission of a 10-MeV electron beam 
through $0.1 \mathrm{~mm}$ of lead. In each of these figures two sets of results are shown, one for a lead foil only, and the other for a lead foil followed by a layer of material equivalent to $100 \mathrm{~cm}$ of air. These results are representative of those obtained for other foil thicknesses and beam energies.

Energy spectra of transmitted electrons for a 10-MeV beam incident on a $0.1-\mathrm{mm} \mathrm{Pb}$ foil are given in Fig. 2. This figure also shows the shift towards lower energies and the broadening of the spectrum that occur when electrons also pass through $100 \mathrm{~cm}$ of air after going through the foil. In Table 1, the mean energy loss, the most probable energy loss and the full width at half maximum of the spectrum are given for other combinations of beam energy and lead target thickness (always with an additional $100 \mathrm{~cm}$ of air). Also shown in Table 1 are results from Landau's ${ }^{20}$ theory which takes into account the fluctuations of collision losses, but includes neither bremsstrahlung losses nor the lengthening of the electron paths in the target due to multiple elastic scattering. It can be seen that in spite of the omission of these two effects the Landau theory provides a rather good estimate of the most probable energy loss, whereas it predicts a full width at half maximum about one third smaller than the Monte Carlo values.

Figure 3 shows a plot of the angular distribution of the transmitted electrons (per unit solid angle) vs. the square of the deflection angle. On this semi-logarithmic plot, the calculated points can be accurately fitted by a straight line, which indicates that in very good approximation the angular distribution is Gaussian, i.e. proportional to $\exp \left(-\theta^{2} / \alpha^{2}\right)$. This approximation holds for $\theta \leqslant 20^{\circ}$, but tends to break down for larger angles. Values of the parameter a calculated for various combinations of beam energy and lead foil thickness are given in Table 2. These results are close to the root-mean-square angles predicted by multiple-scattering theories in the small-angle approximation, as tabulated, for example, by Brahme. ${ }^{21}$ 
The energy spectra and angular distributions of transmitted bremsstrahlung photons are shown in Figs. 4 and 5. The angular distribution is not Gaussian, and is more concentrated in the forward direction than that of the electrons. Figure 6 shows the fraction of the incident beam energy that is transmitted through the foil and reaches the phantom, either in the form of electrons or of secondary bremsstrahlung. These two quantities are shown as functions of the cone half-angle $\theta_{c}$ which is equal to the arctangent of the ratio of the field-size radius $r_{F}$ to the foil-phantom distance $s$. It can be seen that the relative contribution of bremsstrahlung from the foil to the energy deposited in the phantom is greatest when the field size is small.

\section{Elementary Absorbed Dose Distributions}

Assuming a water phantom occupying the region $z \geqslant 0$, and monoenergetic, point-monodirectional electron beams incident along the z-axis, absorbed-dose distributions in the phantom were calculated by the Monte Carlo method for nine beam energies $(1,5,10,15,20,30,40,50$ and $60 \mathrm{MeV})$. These distributions were obtained as functions of the depth $z$ in the phantom and the radial distance $\rho$ from the $z$-axis. An illustrative set of results for a $20-\mathrm{MeV}$ beam is shown in Fig. 7. The histogram in the upper right-hand corner is the depth-dose distribution. The other six histograms are radial distributions in various depth intervals, and show the fractions of the energy deposited in ring-shaped layers around the z-axis. In Fig. 7, depths and radial distances are expressed in units of the electron range $r_{0}$. It has been found that through this scaling the explicit dependence of the absorbed-dose distribution on the incident beam energy is greatly reduced, so that interpolation with respect to initial beam energy is facilitated.

As a check on the validity of the method, some Monte Carlo calculations were also made for an acrylic plastic (Lucite) medium for comparison with experimental absorbed-dose distributions obtained without a scattering foil or 
collimator. Figure 8 shows a comparison with depth-dose curves measured by Harder and Schul $\mathrm{z}^{22}$ at $21.2 \mathrm{MeV}$ and $62 \mathrm{MeV}$ in Lucite, under broad-beam conditions (actually with a very narrow beam and a very broad detector). Figure 9 shows a comparison with radial dose distributions measured by Li1licrap, Wilson and Boag ${ }^{23}$ at depths of 1 and $2 \mathrm{~cm}$ in a Lucite medium irradiated with a non-divergent 10-MeV, 2.5-mm diameter beam. The quantity plotted is the radial dose profile, i.e. the dose as a function of distance from the beam axis, normalized to unit central-axis dose. The overall agreement between calculated and measured results in Figs. 8 and 9 is good.

Additional Monte Carlo calculations were done in the course of the present work to obtain absorbed-dose distributions in water from the bremsstrahlung emitted by the scattering foil. These were done for pointmonodirectional beams, assuming photon spectra obtained in the preceeding foil-transmission calculations.

\section{Combination of Elementary Absorbed Dose Distributions}

In the last stage of the calculation, the electron beam reaching the phantom after passage through the foil (and intervening air) was considered to be a weighted superposition of different elementary, monoenergetic, monodirectional beams with different energies, obliquities and points-of-entry into the phantom. The weighting factors were obtained from the foiltransmission calculations. The absorbed-dose distribution from the composite beam, as a function of depth and radial distance from the beam axis, was correspondingly obtained by summing over the weighted elementary absorbed-dose distributions previously obtained. By limiting the summation over obliquities to certain cones around the beam axis, the effect of collimation to finite field size was simulated. However, this treatment did not take into account 
the effect of electron scattering from a collimator. A similar superposition over obliquities and points-of-entry was also applied to the dose contribution due to bremsstrahlung from the foil.

The application of the superposition procedure required the prior smoothing of the Monte Carlo results for elementary absorbed dose distributions, and extensive interpolation with respect to beam energy and the two spatial variables. The computations were found to be moderately difficult for the evaluation of the central-axis depth dose, and quite laborious for the evaluation of the radial dose profile, i.e. the dependence of absorbed dose on the distance from the beam axis. The resulting numerical uncertainties in the latter case may have been as great as $5 \%$ in some cases, and there is a need for improving the speed as well as the accuracy of the superposition calculations.

\section{Central-axis Depth Dose}

Calculated central-axis depth-dose curves are compared in Fig. 10 for 10-MeV beams. Curves are given for a monoenergetic, non-divergent beam with a 20-cm diameter (no foil case), and for beams that have passed through a lead foil $(0.1 \mathrm{~mm}$ or $0.4 \mathrm{~mm})$ plus $100 \mathrm{~cm}$ of air. The presence of a scattering foil raises the entrance dose relative to the peak dose by several percent.

Changing the foil thickness leaves the depth-dose curves practically unchanged near the surface, but results in a significant shift at depths beyond the peak. Depth-dose curves for 30-MeV beams are compared in Fig. 11. Results are given for a monoenergetic non-divergent beam with a $20-\mathrm{cm}$ diameter (no foil case), and for beams that have passed through $0.4 \mathrm{~mm}$ of lead and $100 \mathrm{~cm}$ of air, and that have been collimated to fields with $20-\mathrm{cm}$ or $5-\mathrm{cm}$ diameters. Again the presence of the foil raises the entrance dose relative to the peak dose. The change of field size from 5 to $20 \mathrm{~cm}$ leaves the depth-dose curves 
unchanged from the surface to the peak at $3.5 \mathrm{~cm}$; at greater depths the collimation to a $5-\mathrm{cm}$ diameter makes the depth-dose curve fall off much more rapidly than that for a $20-\mathrm{cm}$ diameter because of increased out-scattering.

Figure 12 shows the estimated contribution to the central-axis depth-dose made by bremsstrahlung from the scattering foil. Results are given for 10-, 20 - and $30-\mathrm{MeV}$ beams that have passed through $0.4 \mathrm{~mm}$ of lead plus $100 \mathrm{~cm}$ of air. The relative bremsstrahlung contribution is small near the surface, then rises and reaches a plateau of several percent at small and intermediate depths; finally it becomes predominant at depths comparable with the electron range.

Calculated central-axis depth-dose curves are compared in Fig. 13 with experimental results of $H$. Svensson (private communication). The experimental conditions for the cases selected for comparison were: (a) 41-MeV beam, 0.125-mm aluminum window, 0.6-mm lead foil; (b) 22.5-MeV beam, 0.2-mm aluminum window, 0.17-mm uranium foil; in both cases the field size was unlimited and the foil-to-phantom distance in air was $100 \mathrm{~cm}$. Comparison calculations are available for the following conditions: (a) 40-Mev beam, 0.6-mm lead foil plus $100 \mathrm{~cm}$ of air, field diameter $20 \mathrm{~cm}$; (b) 22.5-MeV beam, $0.2-\mathrm{mm}$ aluminum window, $0.17-\mathrm{mm}$ uranium foil plus $100 \mathrm{~cm}$ of air, field diameter $40 \mathrm{~cm}$. The calculated results at $40 \mathrm{MeV}$ have been adjusted to $41 \mathrm{MeV}$ by "stretching" the depth scale through multiplication by the ratio of range values at 41 and $40 \mathrm{MeV}$. The agreement between experiment and calculation is very good close to the entrance surface and at great depths near the end of the electron range. At intermediate depths, beyond the peak, the experimental and calculated curves appear to be shifted somewhat with respect to each other; the direction of shift is different for the two beam energies. 


\section{Radial Dose Profiles}

Radial dose profiles are shown in Fig. 14 for a 10-MeV beam that has passed through a $0.1-\mathrm{mm}$ lead foil and $100 \mathrm{~cm}$ of air, and has been collimated to a $5-\mathrm{cm}$ diameter field. At the sha?low depth of $0.6 \mathrm{~cm}$, the radial dose profile is rather flat out to $2.1 \mathrm{~cm}$, and actually has a peak value of 1.02 at a radial distance of $1.5 \mathrm{~cm}$ from the beam axis. This very small rise above unity, appears to be the result of the angular divergence of the incident beam. The radial profiles at the other depths shown $(1.2,2.4$ and $3.2 \mathrm{~cm}$ ) reflect the broadening of the beam with increasing depth due to multiple scattering. The value of the radial profile at the edge of the beam (distance of $2.5 \mathrm{~cm}$ from the axis) is rather independent of the depth, and lies between 0.45 and 0.5 .

In Fig. 15 calculated radial dose profiles are compared with recent experimental results of Svensson. The cases selected for comparison were the same as those in the central-axis depth-dose comparisons in Fig. 13. The profiles are compared at the depths where the corresponding central-axis depth-dose curves peak. The experimental field size was unlimited, whereas in the calculation it was 1 imited to a $20-\mathrm{cm}$ diameter at $41 \mathrm{MeV}$ and a $40-\mathrm{cm}$ diameter at $22.5 \mathrm{MeV}$. In view of this difference one must limit the comparison to radial distances smaller than the field radius assumed in the calculation. The agreement is very good at $41 \mathrm{MeV}$, but not as close at 22.5 MeV where the experimental profile falls off somewhat more rapidly than the calculated profile.

\section{Concluding Remarks}

A method has been developed for calculating the spatial distribution of absorbed dose from high-energy electron beams. The new aspect of the method is that it takes into account not only the multiple scattering of the electrons 
in the irradiated phantom, but also the effects of $(a)$ the energy degradation and angular diffusion of the electron beam by a scattering foil, and (b) the limitation of the beam to finite field size. The approach used involves separate Monte Carlo calculations of foil transmission and beam penetration into a phantom, and the subsequent application of these Monte Carlo results, by suitable combination, to specific scattering foil-phantom configurations. Results are presented for some typical cases, for lead scattering foils and electron beams with energies up to $40 \mathrm{MeV}$, in the form of central-axis depth-dose curves, and of dose profiles as functions of the radial distance from the beam axis. A few comparisons indicate reasonably good, but not perfect, agreement with measured absorbed-dose distributions. In view of the considerable amount of computational effort required, it does not yet appear practical, in the present stage of development of the method, to treat routinely the numerous cases that may be of interest in practice, involving different foil materials and thicknesses, foil-to-phantom distances, field sizes, etc. We believe that for the time being the theoretical approach may be most valuable in providing (a) an improved understanding of the influence of various factors on the absorbed-dose distributions, and (b) independent confirmation of measured results.

Acknowledgements. This work was supported by the Office of Naval Research, the Department of Energy (Division of Biomedical and Environmental Research) and the National Cancer Institute. We are grateful to Dr. H. Svensson for providing us with unpublished results of his recent absorbed-dose measurements. 


\section{REFERENCES}

[1] L. V. Spencer, Phys. Rev. 98, 1597 (1955).

[2] L. V. Spencer, Energy Dissipation by Fast Electrons, Nat. Bur. Standards, Monograph 1 (1959).

[3] N. D. Kessaris, Calculated absorbed dose for electrons, Radiat. Res. 23, 630-640 (1964).

[4] N. D. Kessaris, Penetration of high-energy electron beams in water, Phys. Rev. 145, 164-174 (1966).

[5] N. D. Kessaris, Spectra of high-energy electron beams in water, Radiat. Res. 43, 281-287 (1970).

[6] M. J. Berger, Monte Carlo calculation of the penetration and diffusion of fast charged particles, in methods in computational physics, B. Alder, S. Fernbach and M. Rotenbert, Eds., Academic Press, N.Y. (1963).

[7] M. J. Berger and S. M. Seltzer, Ann. New York Academy of Sciences, 161, 8 (1969).

[8] M. J. Berger and S. M. Seltzer, Paper presented at the 12th Int. Cong. Radiol. Tokyo, Japan (1969).

[9] H. Sugiyana, Monte Carlo program for photon and electron transport calculations, Bull. Electro-tech. Lab (Ministry of International Trade and Industry, Tokyo, Japan), 34, 126-140 (1970).

[10] S. M. Seltzer and M. J. Berger, Nucl. Instr. Meth. 119, 157 (1974).

[11] J. Rassow, Habilitationsschrift, Ruhr Universitaet (1970).

[12] H. Svensson, Acta Radiol. Ther. Phys. Biol. 10, 631 (1971).

[13] M. Cohen, D. E. A. Jones and D. Green, Eds., Hospital Physicists' Assoc. Central Axis Depth Dose Data for Use in Radiotherapy, Supplement No. 11, Brit. J. Radiology (1972).

[14] P. R. Almond and E. E. deAlmeida, Radiation Therapy with the Electron Beam. Course Manual, Radiological Society of North America (1973).

[15] A. Brahme, G. Hulten and H. Svensson, Phys. Med. Biol. 20, 39-46 (1975).

[16] S. Kramer, N. Suntharalingam and G. F. Zinninger, Eds., High-Energy Photons and Electrons: Clinical Applications in Cancer Management, Wiley, New York (1976).

[17] A. Brahme and H. Svensson, Medical Phys. $\underline{3}, 95-102$ (1976).

[18] F. Wachsmann and G. Drexler, Graphs and Tables for Use in Radiology, Springer, Berlin Heidelberg-N.Y. (1976). 
[19] D. Fehrentz, Habilitationsschrift, U. of Heidelberg (1976).

[20] L. Landau, J. Phys. U.S.S.R. 8 , 201 (1944).

[21] A. Brahme, Report TRITA-EPP-72-17, Department of Electron Physics, Roy. Inst. Technology, Stockholm, Sweden (1972).

[22] D. Harder and H. J. Schultz, Some new Physical Data for Electron Dosimetry, paper given at Europ. Congress of Radiology, Amsterdam (1971).

[23] S. C. Lillicrap, P. Wilson and J. W. Boag, Phys. Med. Biol. 20, 30-38 (1975).

[24] M. J. Berger, S. M. Seltzer, S. R. Doman, and P. J. Lamperti, Stopping power ratios for electron dosimetry with ionization chambers, pp. 589-609, Proc. Symp. on Advances in Biomedical Dosimetry (IAEA-SM-103/39), International Atomic Energy Agency, Vienna, 1975. 



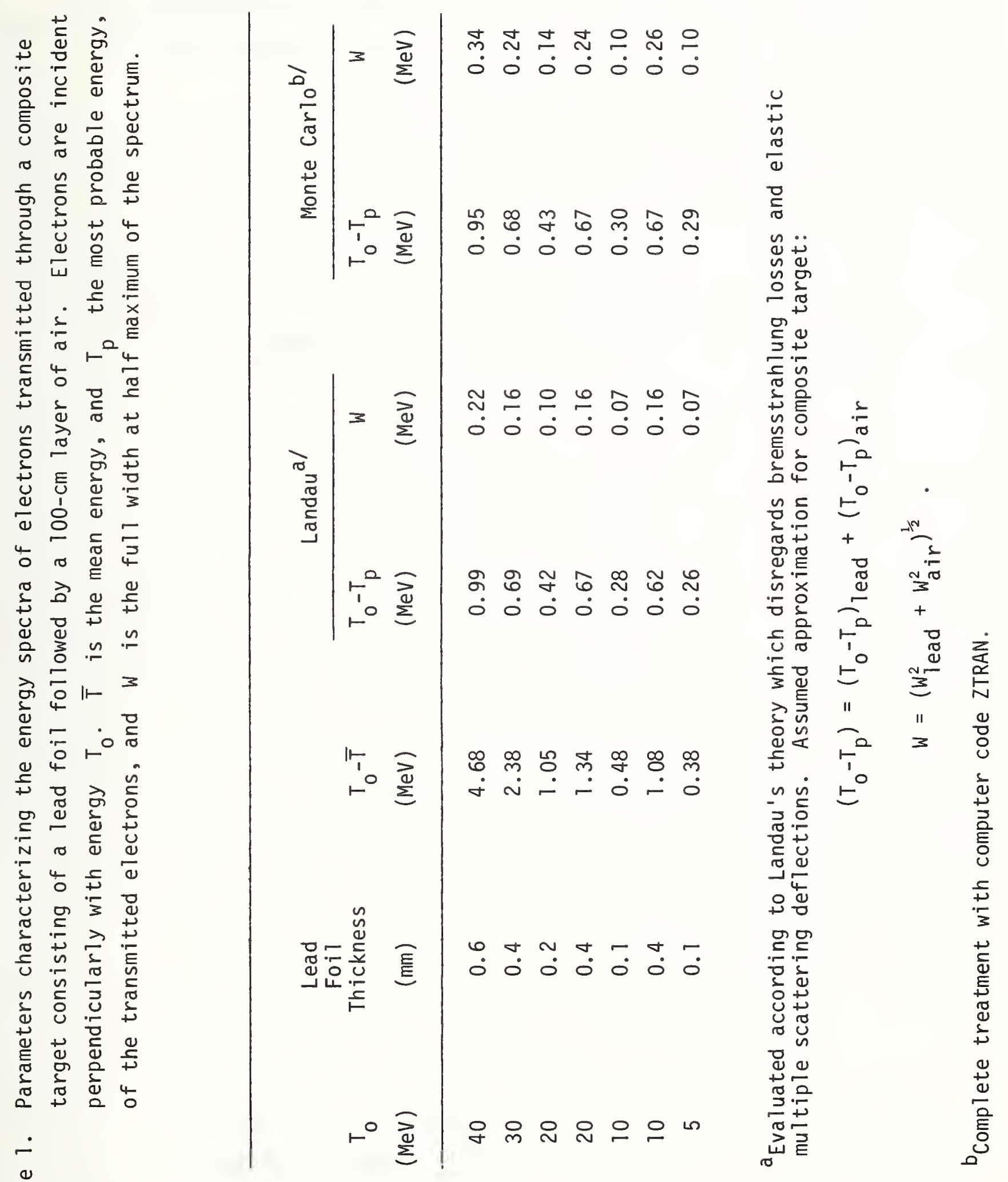

茎 
Table 2. Values of the parameter $\alpha$ in the Gaussian distribution $\exp \left(-\theta^{2} / \alpha^{2}\right)$ used to approximate the angular distribution of transmitted electrons

$\alpha$

\begin{tabular}{cccc} 
& Lead Foil & Foil & Foil Plus \\
$T_{0}$ & Thickness & Only & $100 \mathrm{~cm}$ of Air \\
$(\mathrm{MeV})$ & $(\mathrm{mm})$ & \multicolumn{2}{c}{ (Degrees) }
\end{tabular}

$\begin{array}{rrrr}40 & 0.6 & 9.8 & 9.9 \\ 30 & 0.4 & 10.0 & 10.4 \\ 20 & 0.2 & 10.0 & 10.6 \\ 20 & 0.4 & 14.6 & 14.9 \\ 10 & 0.1 & 13.9 & 15.2 \\ 10 & 0.4 & 29.6 & 30.2 \\ 5 & 0.1 & 24.3 & 26.0\end{array}$




\section{FIGURE CAPTIONS}

Fig. 1 Schematic arrangement of beam, foil, collimator and phantom assumed in calculation.

Fig. 2 Energy spectra $N_{\epsilon}\left(T / T_{0}\right)$ of electrons transmitted through lead foil, or through lead foil plus 100-cm air layer. Electrons with energy $T_{0}$ are assumed to be incident perpendicularly onto the foil, and $T$ is the energy with which the transmitted electrons emerge from the foil, or from the layer of air behind the foil. The spectra include all transmitted electrons that emerge with directions within a cone of $20^{\circ}$ half-angle around the direction of incidence. The curves are normalized such that $\int_{0}^{1} N_{\epsilon}\left(T / T_{0}\right) d\left(T / T_{0}\right)=1$.

Fig. 3 Angular distributions $A_{\epsilon}(\theta)$ of electrons transmitted through a lead foil, or through a lead foil plus a 100-cm air layer. Electrons of energy $T_{0}$ are assumed to be incident perpendicularly onto the foil. The angle $\theta$ is measured with respect to the direction of incidence. The distributions include ail electrons regardiess of the energy with which they are transmitted. The solid and dashed lines are fits to the calculated points. The curves are normalized such that $\int_{0}^{20^{\circ}} \sin \theta A_{\epsilon}(\theta) d \theta=1$.

Fig. 4 Energy spectrum of the bremsstrahiung intensity $\left(k / T_{0}\right) N_{\gamma}\left(k / T_{0}\right)$ from a composite target consisting of a lead $10 i 1$ and a $100-\mathrm{cm}$ air layer. $k / T_{0}$ is the photon energy in units of the incident electron energy $T_{0} \cdot N\left(k / T_{0}\right)$ is the photon number spectrum, and includes al1 photons with directions within a cone of $20^{\circ}$ half-angle with respect to the direction of incidence. The curve is normalized such that $\int_{0}^{1}\left(k / T_{0}\right) N_{\gamma}\left(k / T_{0}\right) d\left(k / T_{0}\right)=1$. The spectral shape is quite similar without the air layer.

Fig. 5 Angular distributions $A_{\gamma}(\theta)$ of bremsstrahiung intensity from a lead foil, or from a lead foil plus a $100-\mathrm{cm}$ air layer, irradiated by electrons of energy $T_{0}$ incident perpendicularly. The distributions include photons of all energies. The curves are normalized such that $\int_{0}^{20^{\circ}} \sin \theta A_{\gamma}(\theta) d \theta=1$. 
Fig. 6 Fractions of the incident electron energy $T_{0}$ that emerge from a lead scattering foil, or from a 100-cm layer of air behind the foil, in the form of transmitted electrons, or in the form of bremsstrahiung photons.

Fig. 7 Energy deposition in water from a point-monodirectional electron beam with energy $T_{0}=20 \mathrm{MeV}$. All distances are expressed in units of the electron range $r_{0}\left(9.21 \mathrm{~g} \mathrm{~cm}^{-2} \mathrm{H}_{2} \mathrm{O}\right)$. The histogram in the upper right hand corner shows energy deposition as a function of scaled depth, $z / r_{0}$, in the form of the dimensionless scaled quantity $\left(r_{0} / T_{0}\right) D\left(z / r_{0}\right)$, where $D$ is energy deposition per electron per unit depth, in $\mathrm{MeV} / \mathrm{g} \mathrm{cm}^{-2}$. The other histograms show, for the six indicated (shaded) depth intervals, the radial distribution of deposited energy, as function of the scaled distance $\rho / r_{0}$ from the beam axis. In these histograms, the area of each bin represents the fraction of the energy deposited in the indicated ring around beam axis. Each histogram is normalized to unit area.

Fig. 8 Comparison of calculated broad-beam depth-dose curves with experimental results of Harder and Schultz ${ }^{22}$, for electron beam energies $T_{0}=21.2$ and $62.0 \mathrm{MeV}$ and an acrylic plastic (Lucite) medium. The quantity plotted is the scaled distribution $\left(r_{0} / T_{0}\right) D\left(z / r_{0}\right)$, where $r_{0}$ is the electron range and $D$ is the energy deposited per electron per unit depth, in $\mathrm{MeV} / \mathrm{g} \mathrm{cm}^{-2}$. In order to make the comparison, we have converted the measured ionization values into absorbed-dose values according to the cavity ionization theory 24 , using depth-dependent Lucite-to-air stopping power ratios $\mathrm{S}_{\text {air }}^{\text {Lucite }}$, which we show as dashed curves.

Fig. 9 Comparison of calculated radial dose profile from beam with small circular cross section with experimental results of Lillicrap, Wilson and Boag ${ }^{23}$. The profiles are expressed as functions of the scaled distance $\rho / r_{0}$ from the beam axis, and are normalized to unit dose on the central axis. 
Fig. 10 Central-axis depth-dose curves for 10-MeV beam that has passed through a lead foil and $100 \mathrm{~cm}$ of air. "No foil" curve is for a monoenergetic beam. Beams are collimated to a diameter of $20 \mathrm{~cm}$.

Fig. 11 Central-axis depth-dose curves for 30-MeV beam that has passed through a lead foil and $100 \mathrm{~cm}$ of air. "No foil" curve is for a monodirectional, monoenergetic beam that has been collimated to a diameter of $20 \mathrm{~cm}$.

Fig. 12 Contribution of bremsstrahlung from scattering foil to the centralaxis depth dose in water. Results are for 10-, 20- and 30-MeV beams passing through a $0.4-\mathrm{mm} \mathrm{Pb}$ foil plus $100 \mathrm{~cm}$ of air.

Fig. 13 Comparison of calculated central-axis depth-dose curves in water with experimental results of Svensson (private communication).

Fig. 14 Calculated radial dose profiles at various depths in a water phantom. Results are for a 10-MeV beam that has passed through a $0.1-\mathrm{mm}$ lead foil plus $100 \mathrm{~cm}$ of air, and has been collimated to a diameter of $5 \mathrm{~cm}$. Dose profiles are normalized to unit dose on the central axis.

Fig. 15 Comparison of calculated radial dose profiles in water with experimental results of Svensson (private communication). Calculated results are for beams collimated to finite field size, whereas experimental results are for uncollimated beams. 



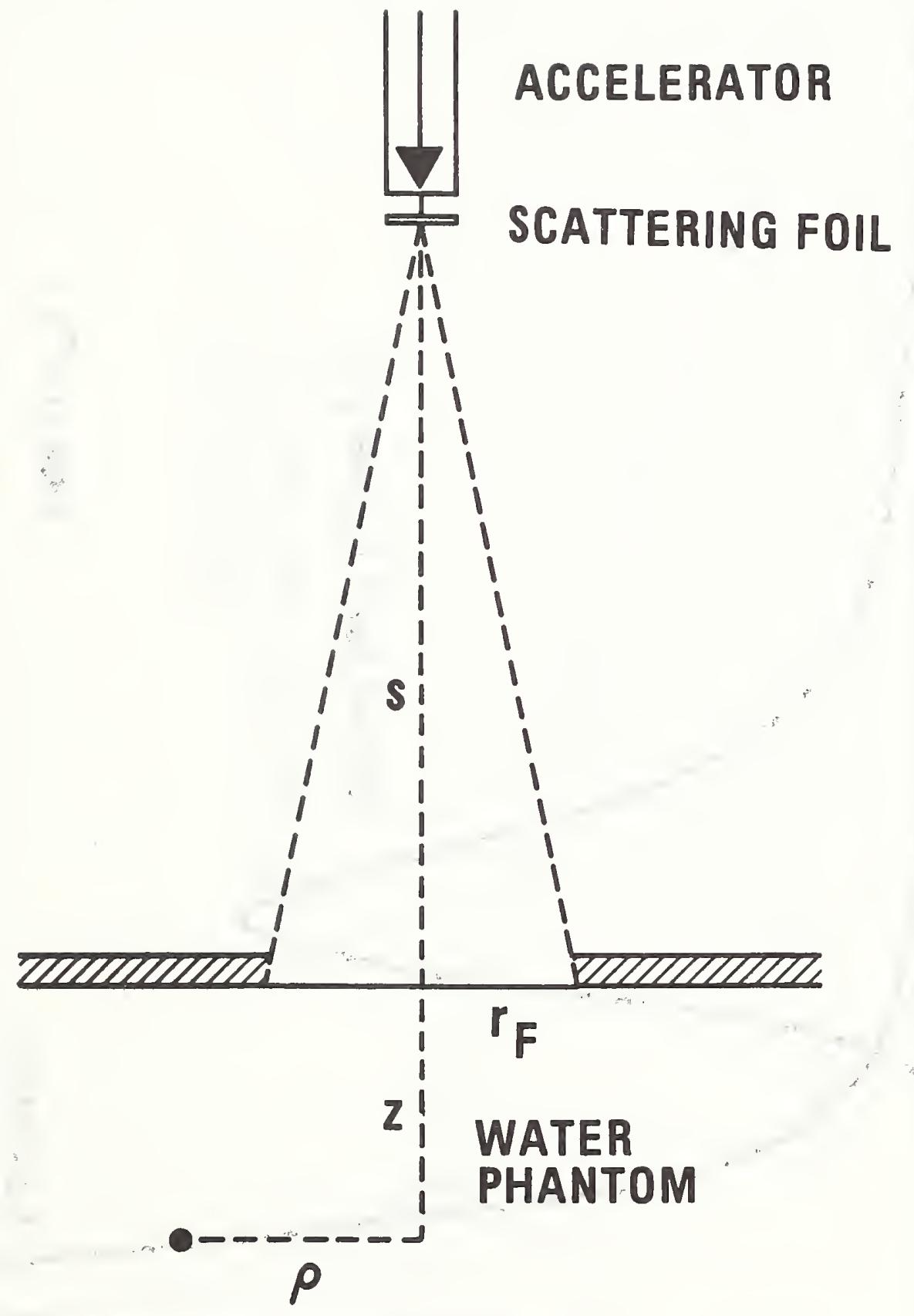

Fig. 1 


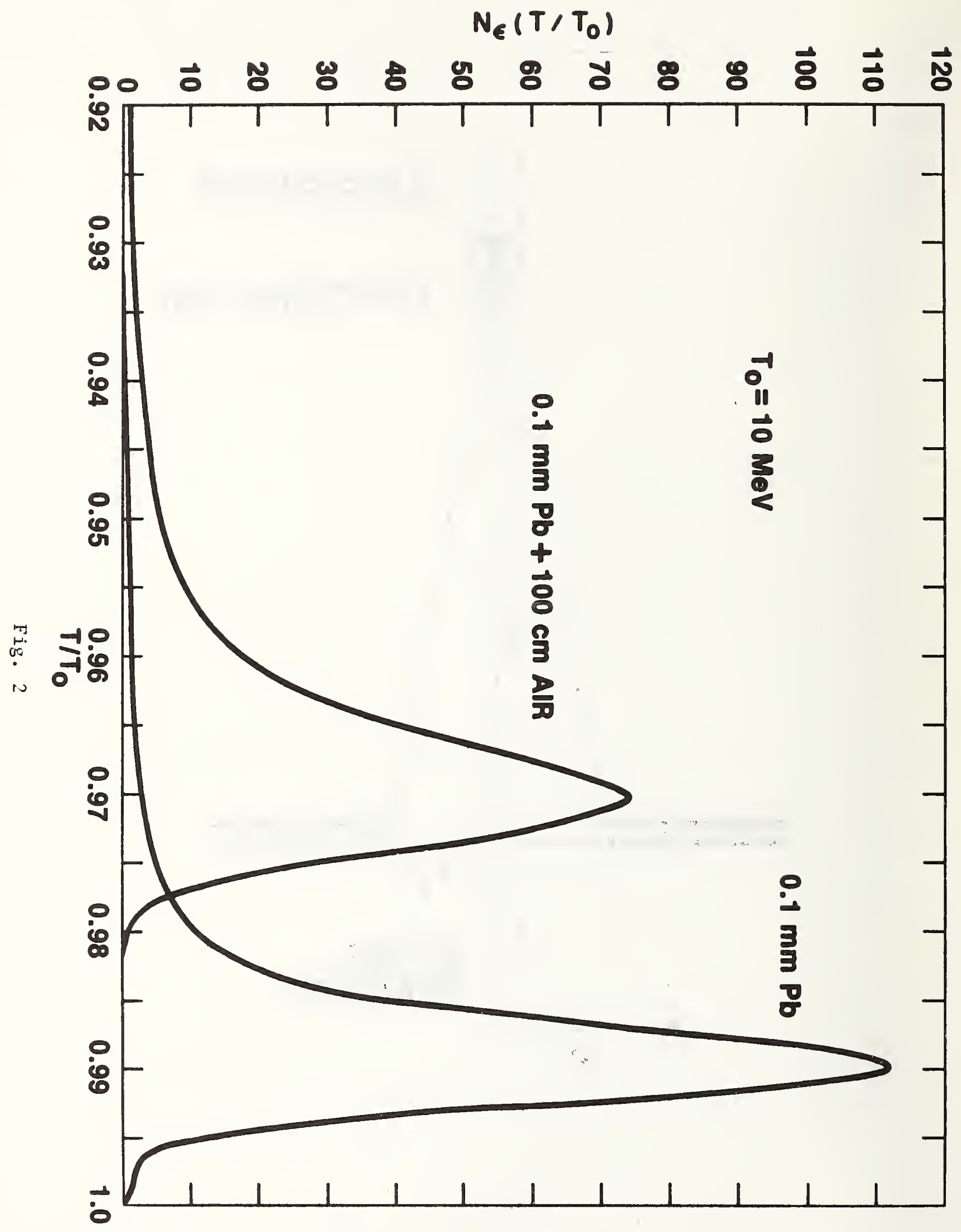




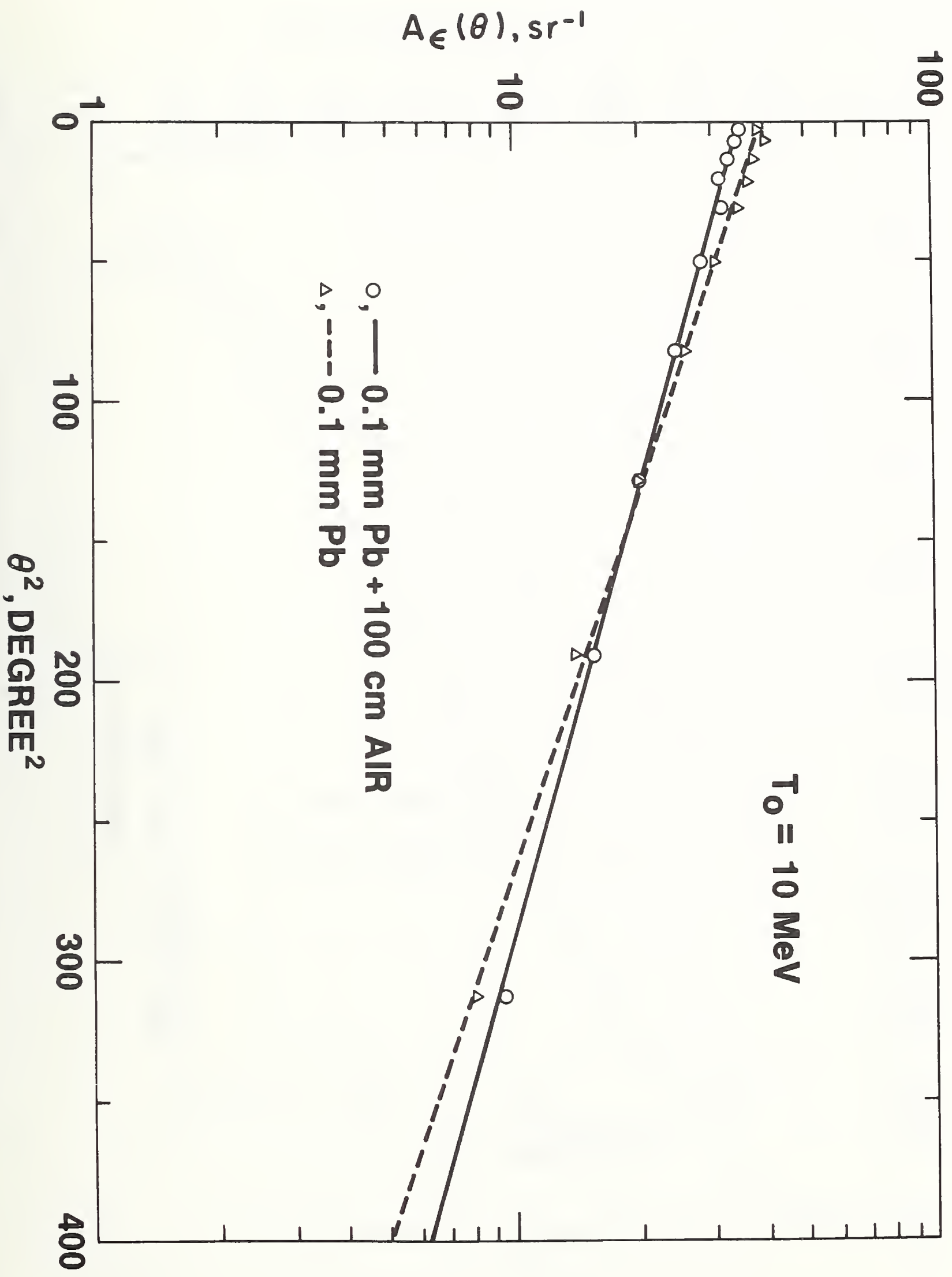




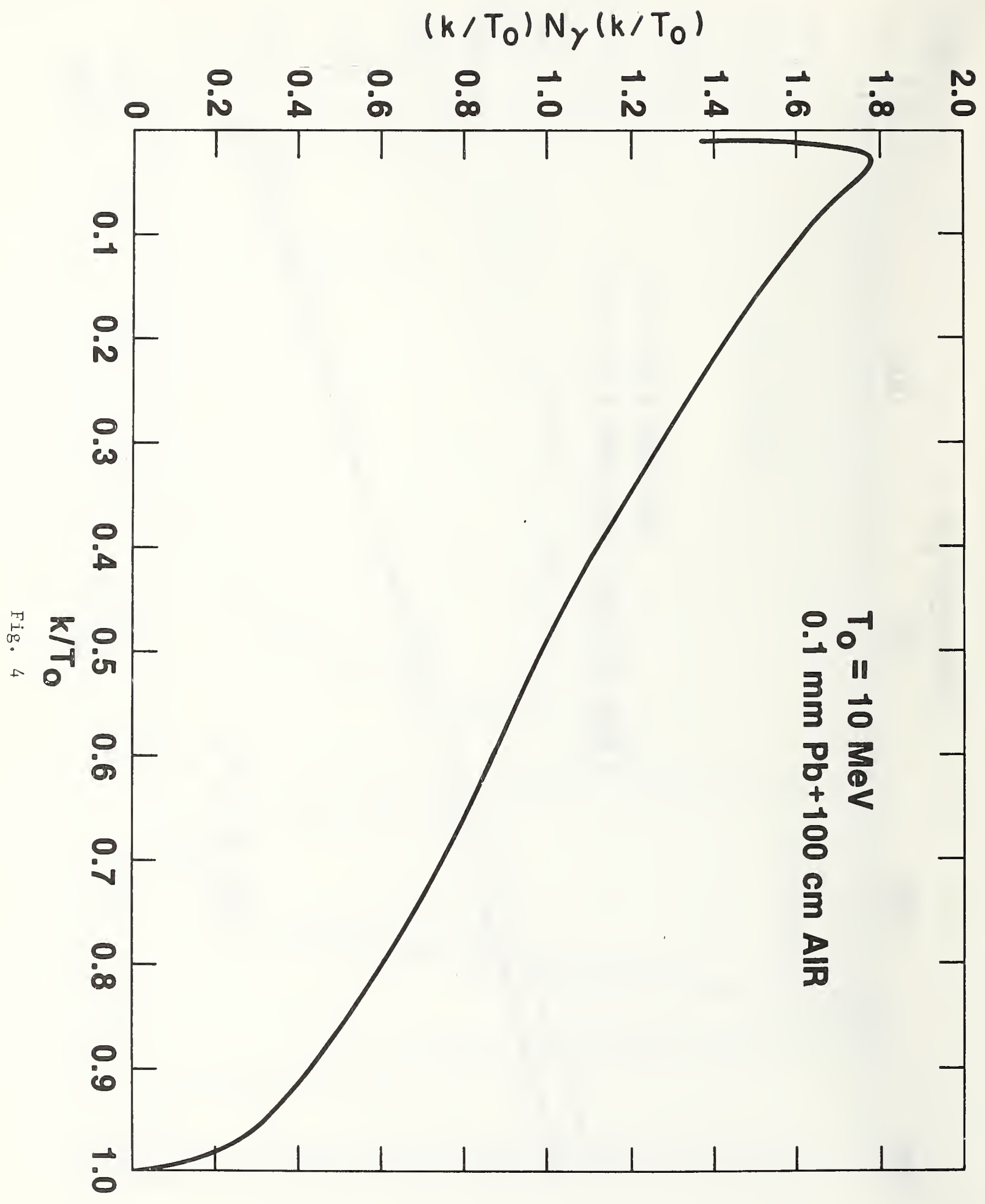




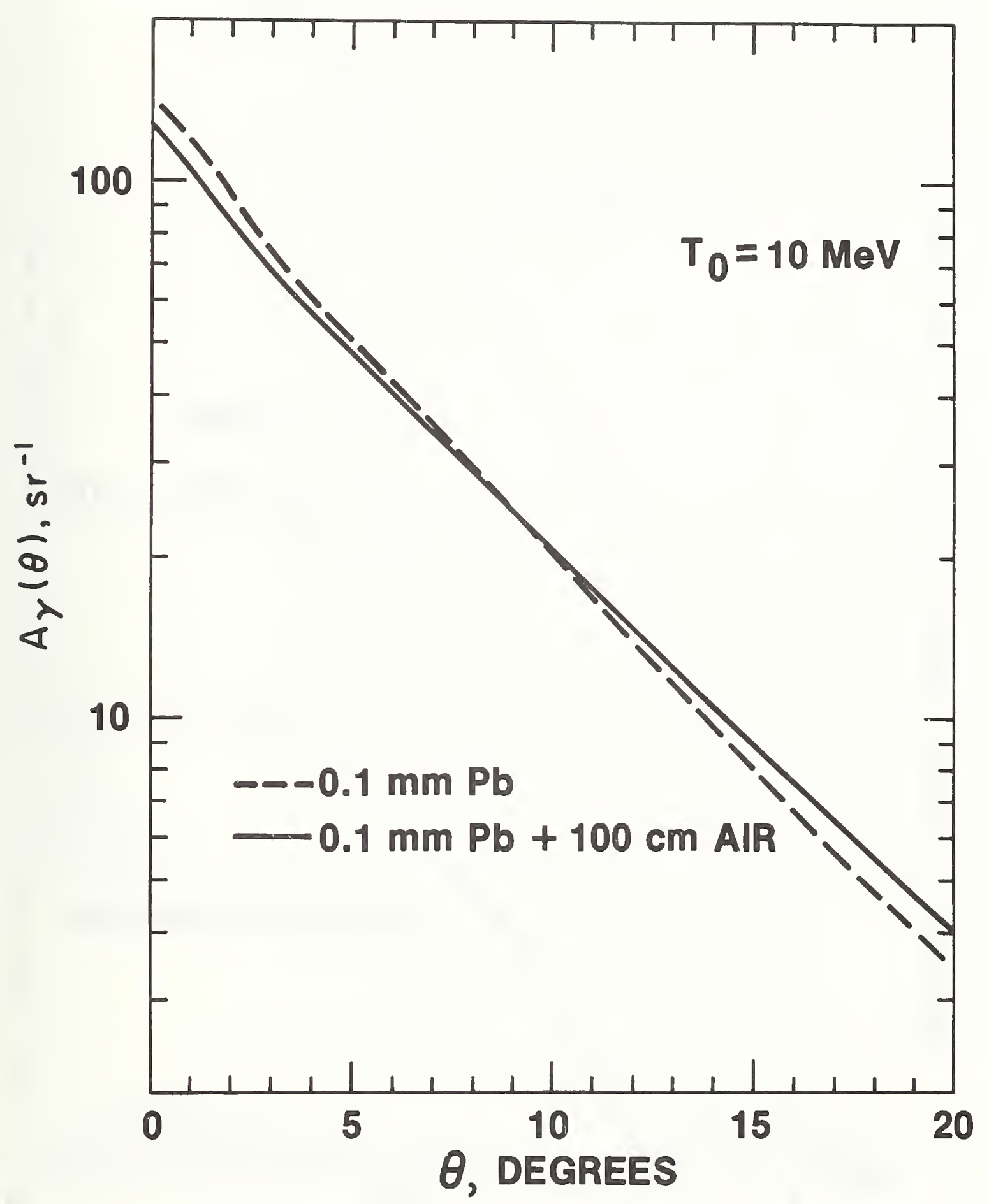

Fig. 5 


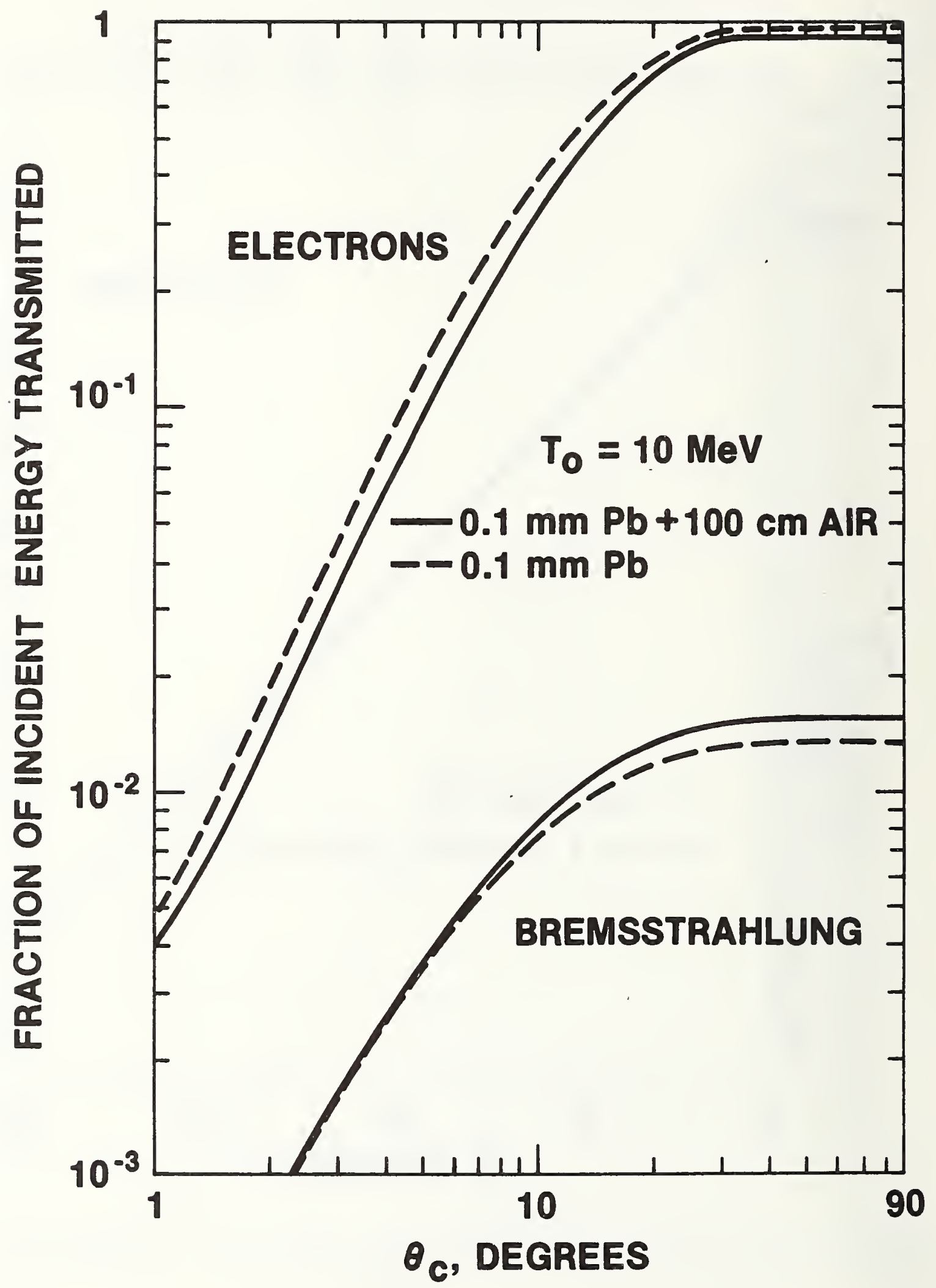

Fig. 6 


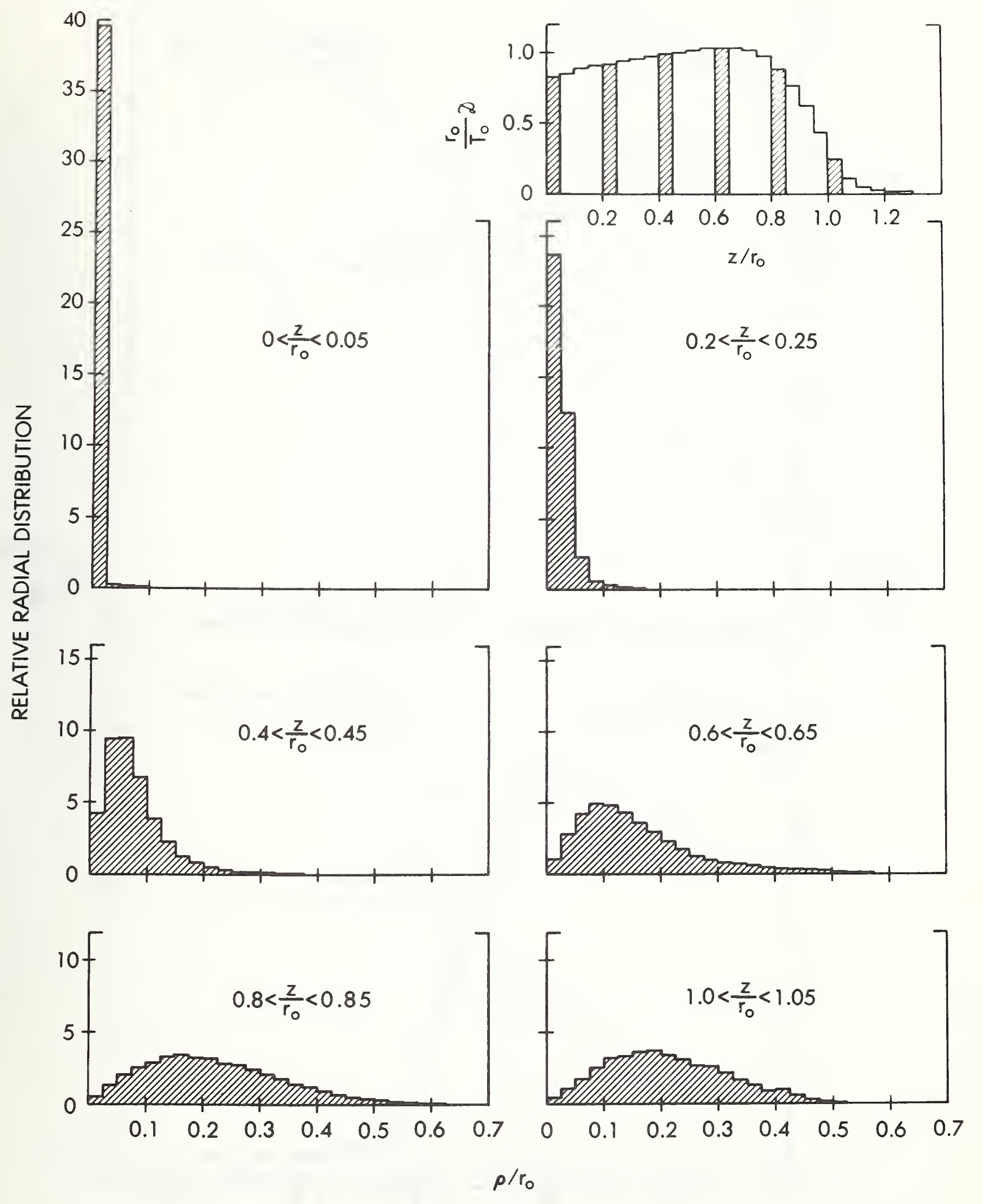

Fig. 7 


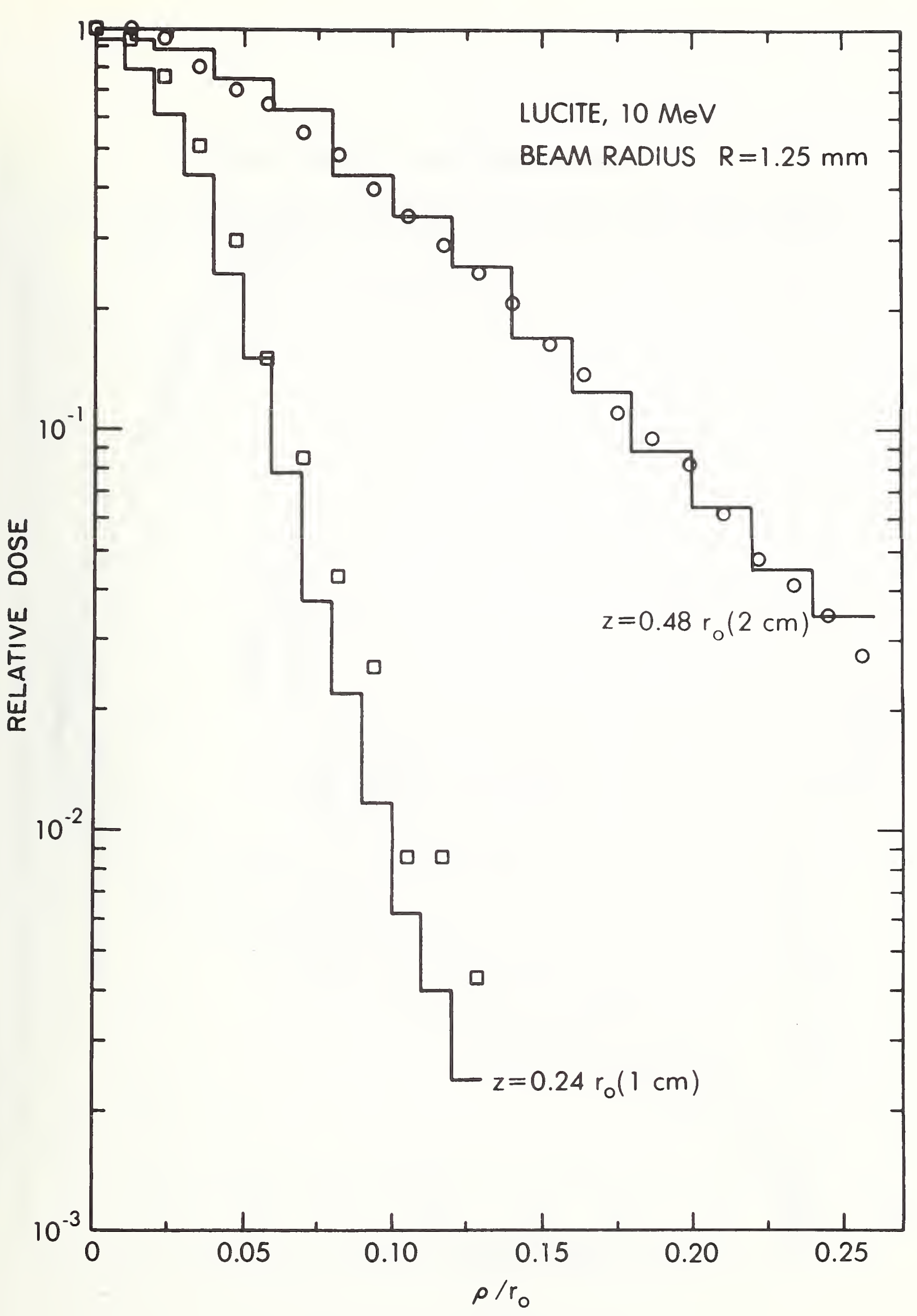

Fig. 9 
RELATIVE CENTRAL - AXIS DEPTH DOSE

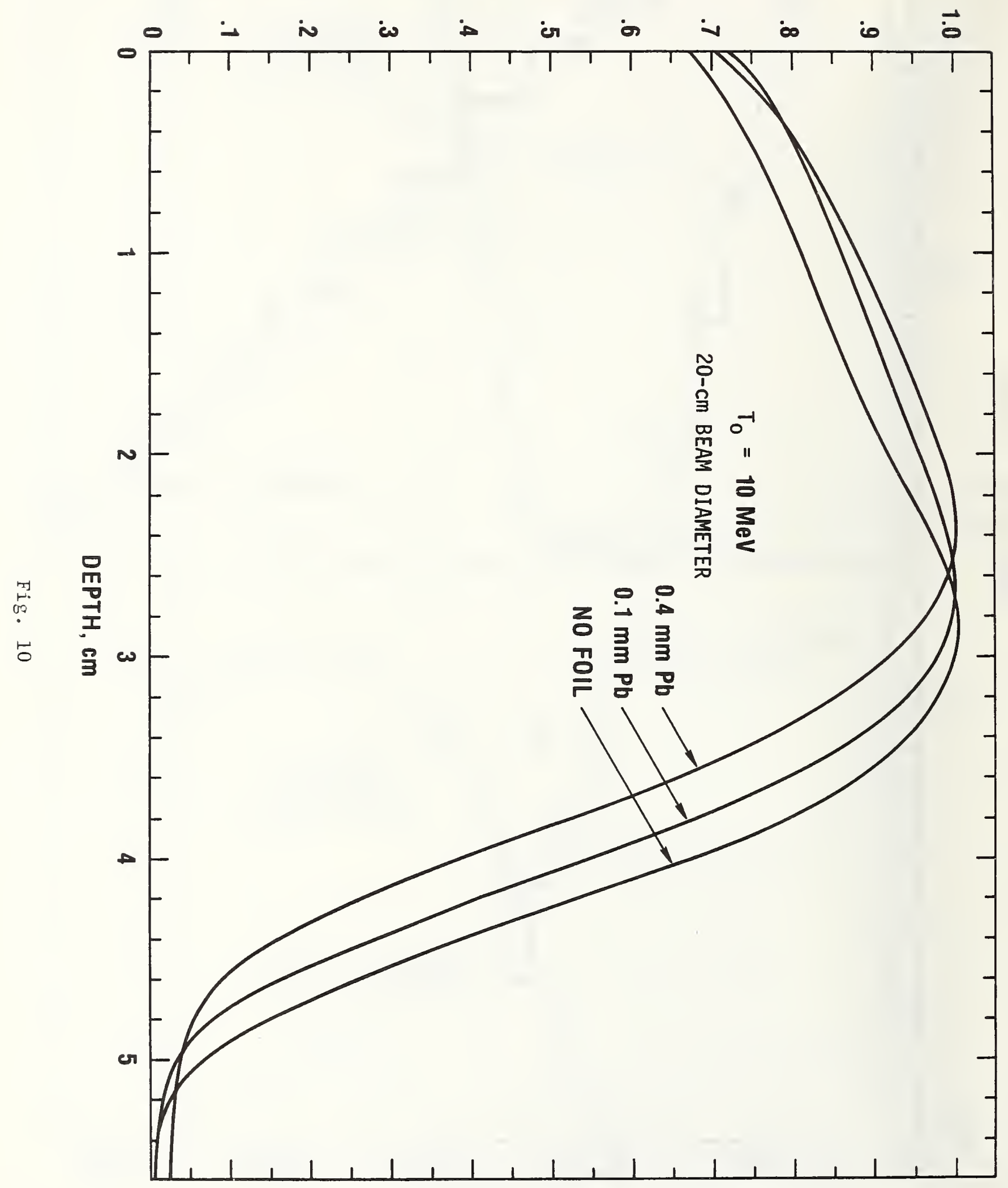




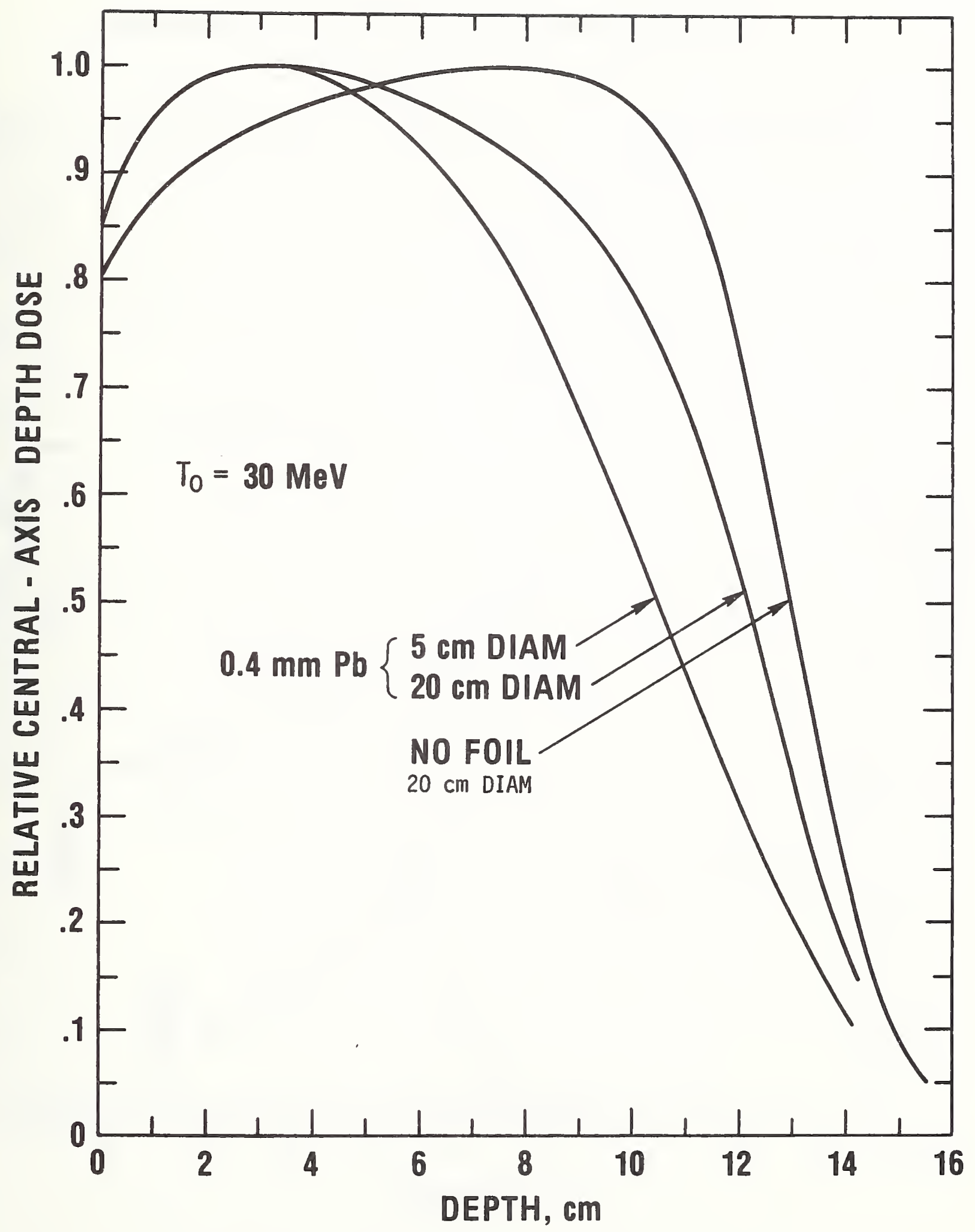

Fig. 11 
BREMSTRAHLUNG CONTRIBUtION, PERCENT

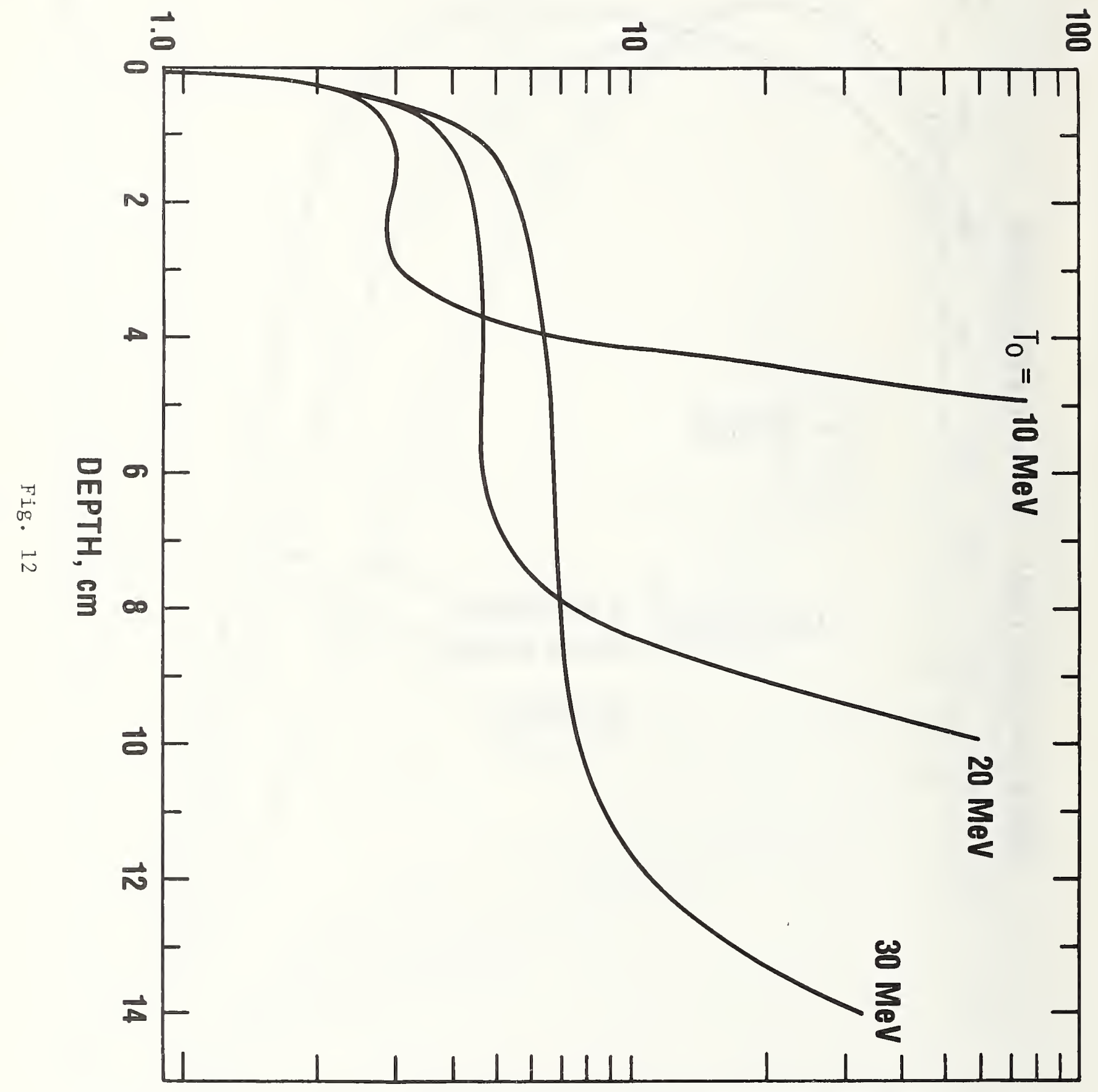




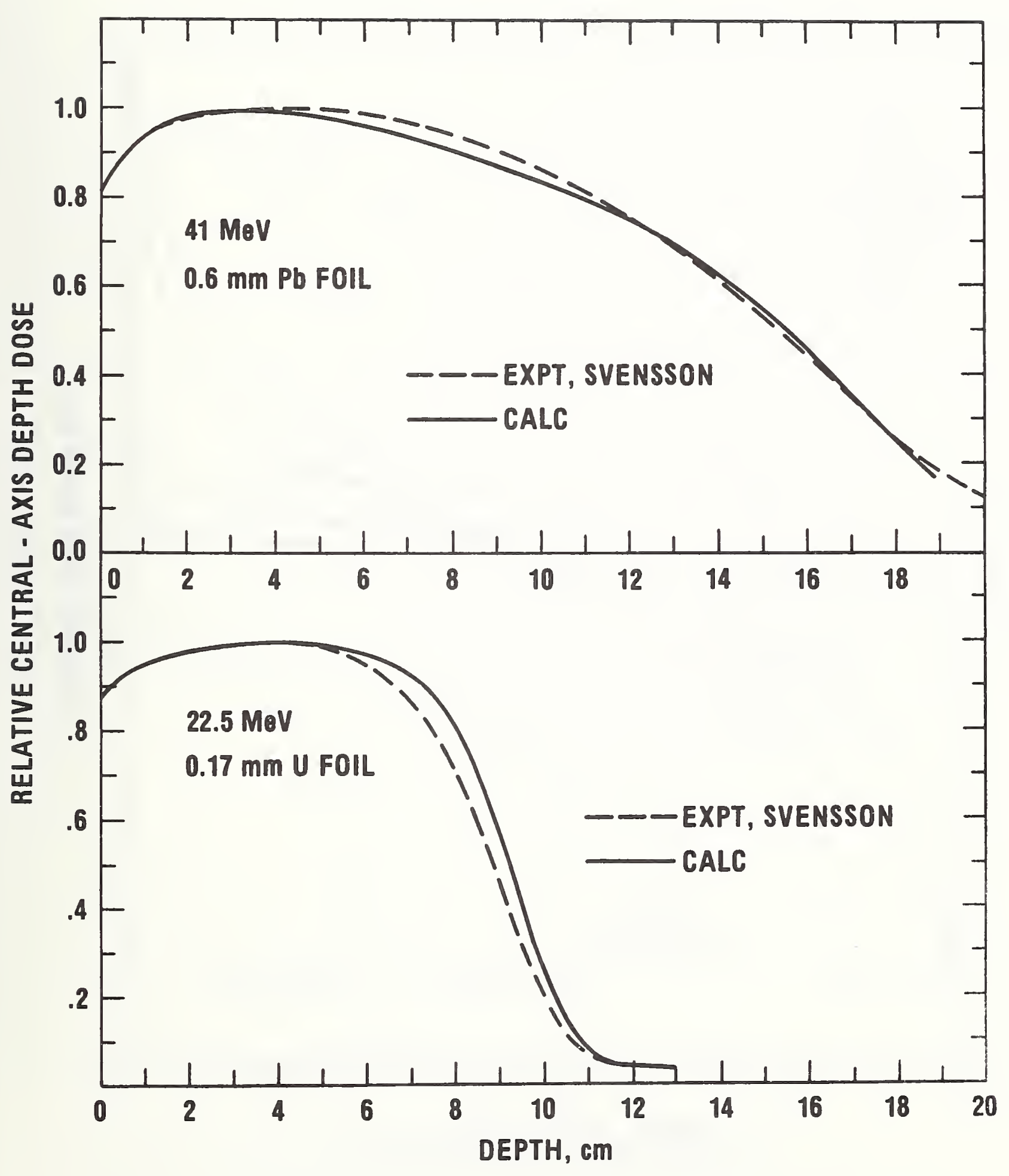

Fig. 13 


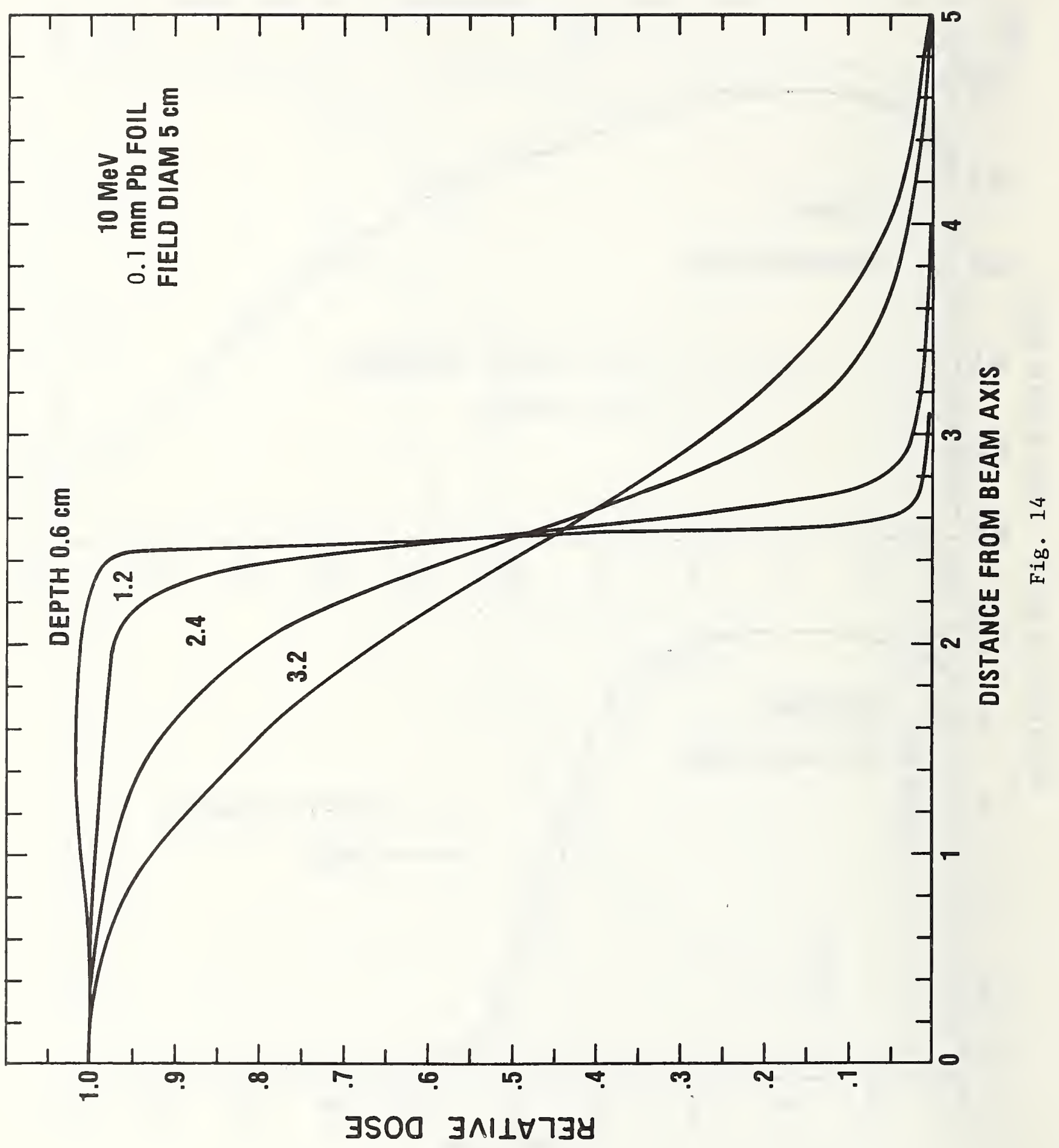




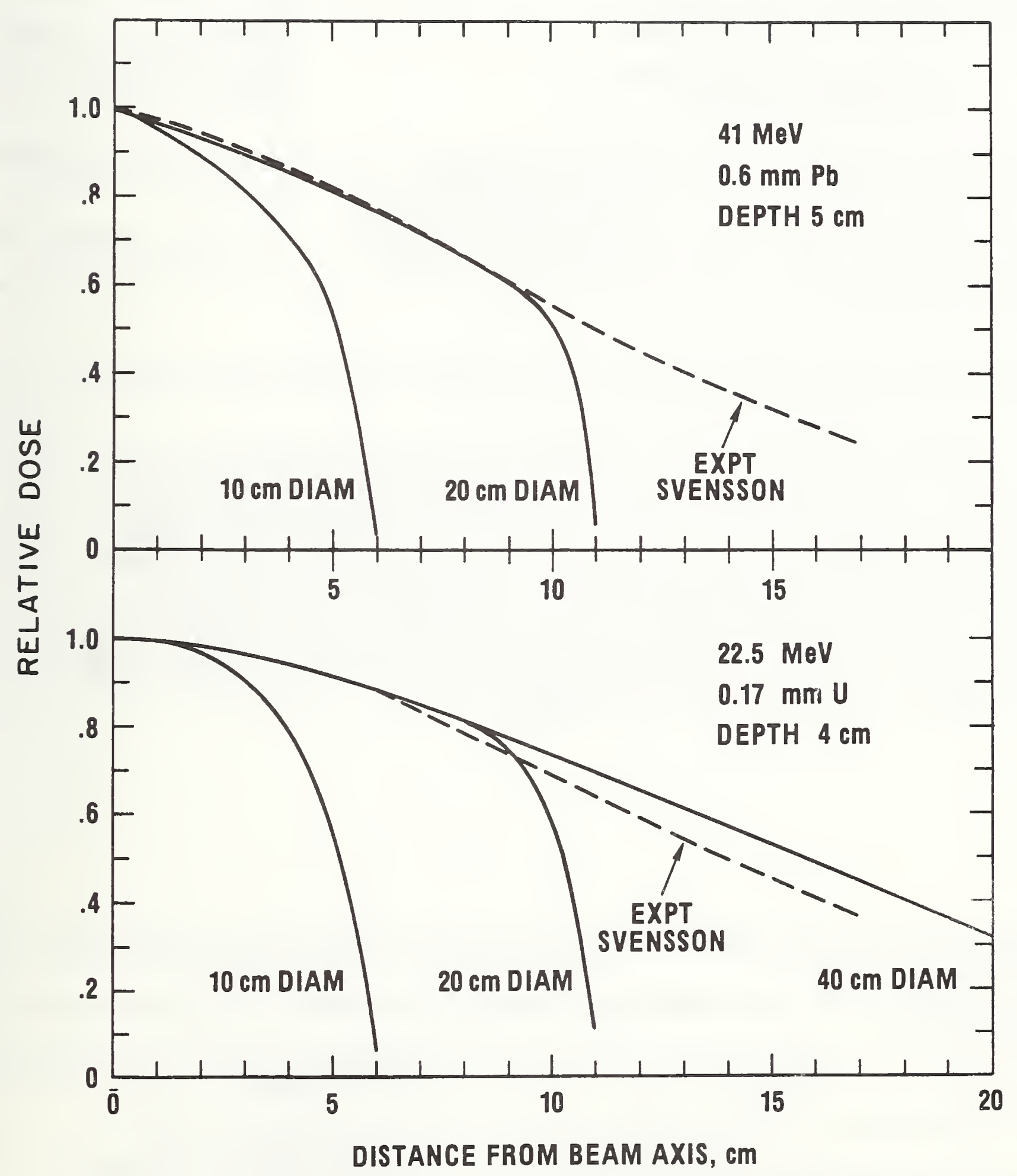




\begin{tabular}{|c|c|c|}
\hline $\begin{array}{l}\text { 1. PUBLICATION OR REPORT NO. } \\
\text { NBSIR 78-1552 }\end{array}$ & $\begin{array}{l}\text { 2. Gov't Accession } \\
\text { No. }\end{array}$ & 3. Recipient's Accession No. \\
\hline \multirow{3}{*}{\multicolumn{2}{|c|}{$\begin{array}{l}\text { 4. TITLE AND SUBTITLE } \\
\text { The Influence of Scattering Foils on Absorbed Dose } \\
\text { Distributions from Electron Beams }\end{array}$}} & \multirow{3}{*}{$\begin{array}{l}\text { 5. Publication Date } \\
\text { 6. Performing Organization Code }\end{array}$} \\
\hline & & \\
\hline & & \\
\hline \multicolumn{2}{|l|}{ 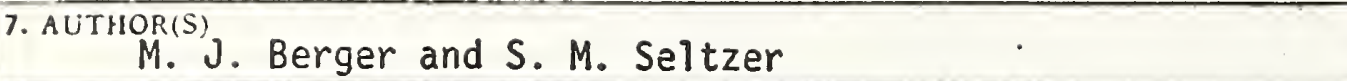 } & 8. Performing Organ. Repose No. \\
\hline \multirow{2}{*}{\multicolumn{2}{|c|}{$\begin{array}{l}\text { 9. PERFORMING ORGANIZATION NAME AND ADDRESS } \\
\text { NATIONAL BUREAU OF STANDARDS } \\
\text { DEPARTMENT OF COMHERCE } \\
\text { WASHINGTON, D.C. } 20234\end{array}$}} & 10. Project/Task/Work thit No. \\
\hline & & $\begin{array}{l}5331439-5337437 \\
\text { 11. Contract/Grant No. }\end{array}$ \\
\hline \multirow{2}{*}{\multicolumn{2}{|c|}{$\begin{array}{l}\text { 12. Sponsurine Organization Name and Complete Address (Street, City, State, ZIP) } \\
\text { Office of Naval Research } \\
\begin{array}{ll}\text { Arlington, Va. } 22217 & \text { Environmental Research } \\
\text { National Cancer Inst.-NIH } & \text { U. S. Department of Energy } \\
\text { Bethesda, Md. 20014 } & \text { Washington, D.C. } 20545 \\
\end{array}\end{array}$}} & $\begin{array}{l}\text { 13. Type of Reporr \& Period } \\
\text { Covered }\end{array}$ \\
\hline & & 14. Sponsoring Agency Code \\
\hline
\end{tabular}

15. SUPPLEMENTARY NOTES

16. ABSTRACT (A 200 word or less factual summary of most significant in formation. It document includes a significant bibliography or literature survey, mention it here.)

This paper presents results of transport calculations pertaining to the distribution of absorbed dose in a water phantom irradiated with high-energy electrons. The electron beam, initially monoenergetic and monodirectional, was assumed to pass through a lead scattering foil and $100 \mathrm{~cm}$ of air before reaching the phantom. The principal aim of the calculation was to determine the energy spread and angular divergence of the electron beam due to its passage through the lead foil and air, and to estimate the resulting modification of the absorbed-dose distribution in the phantom. Also included in the calculation were the efiects of beam collimation to finite field size, and the contribution to the absorbed dose from secondary bremsstrahlung generated in the scattering foil as well as in the phantom.

17. KEY WORDS (six to twelve entries; alphabetical order; capitalize only the first letter of the first key word unless a proper name; separated by semicolons)

Absorbed dose; central axis depth dose; collimation; electrons; radial dose profile; scattering foil.
18. AVAILABIL.ITY
[x Unlimited

[ For Official Distribution. Do Not Release to NTIS

[ Order From Sup. of Doc., U.S. Government Printing Office W'ashington, D.C. 20102, SD C.at. No.C.13

[ Order From National Technical Information Service (NTIS) Springficld, Virginia 22151

\begin{tabular}{|l|l|}
\hline $\begin{array}{l}\text { 19. SECURITY CLASS } \\
\text { (THIS REPURT) } \\
\text { X }\end{array}$ & 21. NO. OF PAGES \\
UNCL ASSIF IED & \\
\hline $\begin{array}{l}\text { 20. SECURITY CLASS } \\
\text { (THIS PAGE) }\end{array}$ & 22. Price \\
UNCLASSIIIED & \\
\hline
\end{tabular}



